\title{
La mondialisation, autrement dit
}

Mouloud Boukala

\section{OpenEdition}

\section{Journals}

Édition électronique

URL : https://journals.openedition.org/pa/1884

DOI : 10.4000/pa.1884

ISSN : 2273-0362

\section{Éditeur}

Université Lumière Lyon 2

\section{Édition imprimée}

Date de publication : 1 janvier 2005

Pagination : 59-63

ISBN : 1634-7706

ISSN : 1634-7706

\section{Référence électronique}

Mouloud Boukala, «La mondialisation, autrement dit », Parcours anthropologiques [En ligne], 5 | 2005, mis en ligne le 06 juillet 2021, consulté le 21 juillet 2021. URL : http://journals.openedition.org/pa/ 1884 ; DOl : https://doi.org/10.4000/pa.1884 


\title{
LA MONDIALISATION, AUTREMENT DIT
}

\author{
"Plus l'angoisse provoquée par un phénomène est grande, moins \\ l'homme semble capable de l'observer correctement, de le penser \\ objectivement et d'élaborer les méthodes adéquates pour le décrire, le \\ comprendre, le contrôler et le prévoir ». \\ Georges Devereux, De l'angoisse à la méthode
}

Cut, cut, cut. Mouvements, secousses, saccades. Cut, cut, cut toutes les trois secondes en cascades. Un montage de plus en plus rapide, un contenu de plus en plus insipide. Tout va trop vite. Voir pose problème, écouter pose problème, comprendre pose problème. Partout l'incompréhensible, nulle part l'intelligible. Voilà la façon de faire et la manière de ne rien dire d'un certain cinéma. Un cinéma propre à tout et bon à rien. Un cinéma où le septième art, cet " art du temps et de l'espace » est réduit à des miettes d'images et à des bribes de temps. Dans ces salles, entre qui veut, voit qui peut, sort qui ose. Ici pas de temps mort, pas de temps qui mord la curiosité du spectateur mais un temps qui dévore toute possibilité d'une rencontre avec l'altérité. Qu'est-ce qui se joue précisément dans ces plans? Un phénomène analogue au mouvement général des choses, l'espace/temps subit un processus de " compression » comme le rappelle David Harvey.

Quelles conséquences pour le cinéma? Le hors champ devient hors sujet. L'image qui logeait de l'autre, du différent, de l'irréductible s'éteint. Le degré zéro de l'altérité est atteint. Une structuration autoritaire des sons et des images est mise en place. Un réalisateur parmi d'autres, Peter Watkins, souligne le cortège de conséquences liées à cette forme narrative organisée :

« Ceci n’a rien à voir avec la complexité, la gamme de possibilités du cinéma ou de la TV en tant que support de création, expression artistique ou type de communication. Cette chose, la Monoforme est devenue LE format obligé structurant tous les films télé et quasiment l'ensemble de la production du cinéma commercial... Ce n'est sûrement pas de la communication. Cela ne permet pas aux spectateurs de participer vraiment. Vous êtes entraînés à travers cette structure narrative monolinéaire par ce formatage frénétique et manipulateur : la Monoforme.

La Monoforme est employée délibérément parce qu'elle ne nous laisse pas le temps de penser ni d'espace pour une participation démocratique permettant une remise en causse, un questionnement. Ceci est un acte délibéré ${ }^{1} »$.

Des millions de spectateurs sont maintenus rivés de manière délibérée à leurs écrans par des agencements de plans dont l'organisation repose uniquement sur l'autorité, la hiérarchie et l'absence de dialogue. Mais comme le rappelait Serge Daney, "l'intelligence, au cinéma, c'est savoir faire semblant de se rallier à la bêtise avant de faire, in extremis, un pas de côté". Faisons ce pas de côté, abandonnons ce cinéma et ses fâcheuses tendances de réduction à l'Un pour un détour qui nous mènera au Brésil, en Sardaigne, en Californie, en Argentine, ... là où il y a de la vigne car, comme le précise Hubert de
Montille $^{2}$, "Où il y a de la vigne, il y a de la civilisation, il n'y a pas de barbarie ". Un détour dont le prix et le charme font la qualité tant du cinéma que de l'anthropologie.

De quoi s'agit-il ? Du quatrième long-métrage de Jonathan Nossiter ${ }^{3}$, Mondovino (2004). Le film s'ouvre sur un ciel bleu avant de découvrir des palmiers et une plage. Les couleurs sont chaudes. Nous sommes dans l'État du Pernambouco dans le Nord-Est du Brésil en compagnie de ramasseurs de noix de coco. Armés d'une sangle, ils parcourent les palmiers d'une hauteur de cinquante mètres. À la montée saccadée des hommes répond la chute continue des fruits. À la question du réalisateur : Peut-on faire du vin de coco ? La réponse fuse : Non, que du jus. Et aussitôt nous quittons les teintes du ciel brésilien pour les colorations plus tamisées d'un coucher de soleil dans les Pyrénées. "L'amour et le plaisir, ce sont des mots qu'on découvre. Mais l'amour et le plaisir, c'est ça qui a mené le monde, qui devrait mener le monde. À 75 ans, je suis surprise par un délire de recherche du plaisir, de partage, de la rencontre... Depuis la mort de mon mari, je me suis mise à planter de la vigne. Et tout cet amour dont je débordais, il est dans la vigne ». Ces mots sont ceux d'Yvonne Hegoburu, propriétaire de 6 hectares de vigne dans le Jurançon, Béarn.

Une minute au Brésil, un peu moins de deux minutes dans les Pyrénées et nous voilà à présent sous un autre ciel. Des collines. Des brebis. Des sons de cloche. Un tonneau avec l'inscription VINO. Une vigne pleine de lumière. Nous sommes à Bosa en Sardaigne, avec un vieux couple attachant et éclairant, Batista et Lina Columbu, propriétaires de deux hectares. Ils reviennent sur la Malvasia, un vin local : "Avant ce n'était pas à vendre. Il fallait venir à Bosa pour en boire. C'était un vin de communauté. Les gens de Bosa offraient la Malvasia en cadeau comme on inviterait un ami à boire le thé. On offrait la Malvasia de Bosa aux amis, aux étrangers... Dans la culture de la Malvasia, il y a une conviction éthique. Un savoir-vivre comme diraient les Français ».

Partons à la découverte de ce savoir-vivre, le raccord se fait à l'aide d'un plan où se détache un avion sur un ciel bien gris. Des panneaux indicateurs: Bordeaux. Nous entendons quelqu'un rire, des bribes de conversation : «il y avait les deux, Gérard Depardieu et Charles Aznavour ». Intérieur voiture, siège arrière, un homme communicatif communique : "Mais vous savez ces journalistes, si on ne leur donne pas des choses qui marquent, ils ne retiennent rien...". De petite taille, vêtu de noir, une barbe fournie, un rire tonitruant, toujours en mouvement, 
consultant en vin pour plus de 100 propriétés dans le monde entier. Voilà Michel Rolland. "Il faut oxygéner». La voiture s'arrête au château Le Gay, à Pomerol. Une conversation s'engage entre la propriétaire, Michel Rolland et le réalisateur :

Michel Rolland : Il faut micro-oxygéner ${ }^{4}$.

Jonathan Nossiter: Mais vous comprenez, Madame, ce qu'est la micro-oxygénation?

Michel Rolland : Non, mais elle s'en fout. Si elle comprenait tout, d'abord je ne serais pas là ! Et puis, si je lui dis " on micro-bulle", elle microbulle. Et puis, si ça marche pas, elle me vire.

La propriétaire : C'est comme ça que ça marche. La spécialité aux spécialistes.

Michel Rolland: De toute façon, le but est très simple. C'est toujours de faire mieux. Il n'y a pas de question à se poser. Si ça doit pas faire mieux, on fait pas.

Jonathan Nossiter : Oui, mais pas tout le monde a les mêmes idées sur ce qui fait mieux un vin.

Michel Rolland: Bien sûr. C'est ce qui fait la diversité. C'est pour cela qu'il y en a autant de mauvais d'ailleurs (rires).

60 Séquence suivante, nous parcourons une départementale bordée de platanes qui nous mène à Aniane dans le Languedoc. Nous retrouvons Jonathan Nossiter arpentant des vignes aux côtés d'un homme âgé au pas ferme et décidé. La pente est prononcée, l'allure est vive, le verbe aussi. Une sentence tombe : "Le vin est mort. Soyons clair, le vin est mort. Il n'y a pas que le vin, il y a les fromages, il y a aussi les fruits... Oui, je dis le vin est mort. Alors, on va essayer de rendre cela clair. Qu'est-ce que le vin? Le vin, pendant des millénaires, c'est une relation presque religieuse de l'homme, à travers la Méditerranée, essentiellement autour de la Méditerranée, une relation presque religieuse avec les éléments naturels. Le sol bien sûr, le sol vivant, le sol sur lequel il n'y a jamais eu de molécules de synthèse et puis le climat ». Voilà Aimé Guibert, propriétaire de 40 hectares à Aniane.

Un rire tranche avec ses paroles: "Il faut oxygéner au maximum ". Michel Rolland poursuit sa tournée tandis que nous retrouvons Aimé Guibert: "Les vins qui ont fait rêver sont toujours des vins qui traversent le temps, qui vous amènent la jeunesse alors qu'il devrait $\mathrm{y}$ avoir les rides et la mort. C'est $1 \%$ des vins au monde! Dans ce domaine des grands vins qui défient le temps, Bordeaux est exceptionnel. Le Bordeaux d'aujourd'hui, je n'en parlerai pas. On a tiré le rideau et on est devant autre chose... Un grand vin, c'est beaucoup d'amour, beaucoup d'humilité, beaucoup de lien avec l'immatériel, avec le sol, avec le temps, avec le climat. C'est un métier de poète de faire du grand vin. Alors maintenant, c'est remplacé par des œnologues. Et des brillants, des séduisants. Comme Monsieur Rolland par exemple. C'est autre chose ".

Pour comprendre cette chose qu'on nomme mondialisation, les dix premières minutes de Mondovino en particulier ainsi qu'un certain cinéma respectueux des spectateurs m'apparaissent indispensables. Le titre de cet article, la mondialisation autrement dit, aurait pu s'intituler comment le cinéma pense la mondialisation? ou plutôt comment le cinéma nous induit-il à penser la mondialisation ? Il s'agit de tenter de cerner ce qu'est la mondialisation et de le faire différemment, c'està-dire en d'autres termes.

De quoi traite Mondovino ? Ni plus ni moins que de la mondialisation des goûts, d'une vanillisation des goûts. Le vin doit désormais être concentré et boisé. Du bois neuf, du chêne plus exactement, naît ce goût vanillé qui s'apprécie immédiatement. Tout commence en 1982, date charnière où s'effectue une authentique révolution dans le monde vinicole : l'apparition des critiques de vin dont le plus important demeure l'américain Robert Parker. Le terme révolution n'est pas tout à fait adéquat, le terme découverte serait plus approprié. À l'instar de Christophe Colomb, Robert Parker a découvert l'Ancien Monde et son or rouge, et dans cette colonisation gustative Hernan Cortes revêt les traits de Michel Rolland.

À eux deux, ils décrètent le temps qu'il fera dans le monde vinicole ${ }^{5}$. Ils sont les "médecins et les psychiatres de la vigne". Ils font le tour du monde comme on fait en clinique, examinent chaque vin dans sa bonne et mauvaise santé et donnent le pouls au marché. Le résultat : des vins techniquement corrects mais sans caractère car, comme l'explique Neal Rosenthal, importateur de vin à New York : " une fois mis le vin dans du bois neuf, il perd son âme. Il a un caractère neutre. Ça devient un vin poupée. Un vin qui n'a pas grandchose à dire ". Si le décor est à présent dressé, les personnages plantés, observons à présent comment le cinéma procède. C'est-à-dire comment le cinéma arrive à faire varier de "façon systématique et contrôlée la focale de son objectif " pour reprendre les termes de Jacques Revel.

Ce faisant, nous découvrirons que les problèmes posés par la mondialisation aux chercheurs en sciences sociales sont des problèmes d'ordre méthodologique, liés à l'articulation d'échelles d'observation. A chaque échelle d'observation correspond en effet un contexte pertinent d'analyse $^{6}$.

Dès lors, comment construire ces contextes ${ }^{7}$ et comment les «brancher»? Comment agencer ces variations d'échelle d'observation? À l'alternative entre le local ou le global, le micro ou le macro, nous devons opposer et observer l'articulation du gros plan et du plan d'ensemble car les questions soulevées par le cinéma sont analogues à celles rencontrées en anthropologie. En d'autres termes, le cinéma tant par ses méthodes, ses dispositifs que son mode d'exposition contribue explicitement à la production d'un certain type d'intelligibilité dans des conditions définies. Un type d'intelligibilité ainsi qu'une toute autre manière de construire scientifiquement la réalité sociale qui sont à même de renouveler l'écriture anthropologique. Qu'est-ce qu'un terrain ? Qu'estce qu'une image ? Comment entre-t-on dans un terrain? Comment sort-on d'un plan?

Les questions posées et exposées ici sont celles d'un chercheur qui tente de décrire, de comprendre des phénomènes dans lesquels des êtres sociaux concrets sont pris dans des relations d'interdépendances spécifiques. Ainsi, dans le présent article, les phénomènes de mondialisation 
seront analysés en termes de possibles. Il s'agira de prendre en compte des destins singuliers confrontés à des choix, d'analyser des conduites, des stratégies tant individuelles que collectives en terme de champs de possibilités. Cette approche permet au chercheur, me semble-t-il, d'éviter deux postures. D'une part, d'être dépêché sur les lieux du sinistre d'un des avions de la Mondialisation Airlines où il tente tant bien que mal de retrouver des boîtes noires et de transcrire les dégâts engendrés par la mondialisation. D'autre part, de partir à la recherche d'un ordre perdu qui mettrait fin à ce "nouveau désordre mondial ».

Revenons à présent à Mondovino et à la galerie de gens mémorables qui nous est présentée. L'un des intérêts majeurs de la démarche de J. Nossiter me paraît être d'inventer incessamment des contextes pertinents, c'est-à-dire des cadres de références qui rendent intelligible cette mondialisation au ras du sol et mettent en lumière les formes du pouvoir au village global. Et de cette invention continue provient l'impression que nous avons de nous figurer cette histoire par nous-mêmes.

Mondovino parvient en effet à filmer le film en train de se faire en interférence avec le réel, à capter le jeu du présent comme rencontre et traversée, tout en s'ancrant dans l'actualité (forum social). Sorte d'utopie du temps réel. C'est bien sûr partiellement une illusion, puisque c'est le résultat de la construction du film. Construction sur laquelle il importe de s'arrêter.

Dans Mondovino, pratiquement aucune scène ne fonctionne isolée du montage. Chaque scène se positionne relativement à plusieurs autres situations du film qui, toujours grâce au montage, se font écho et s'enrichissent les unes les autres. C'est par ce jeu de renvois extrêmement travaillés que le cinéaste fait le tour d'une réalité qui n'est jamais à sens unique mais imbriquée dans une série de contextes. À chaque plan, un niveau de lecture nous est donné et la réalité paraît différente. Et l'un des ressorts les plus puissants de ce film réside dans ce montage, qui relie entre elles ces réalités dans un même système d'interaction aux multiples entrées tout en nous prenant à partie.

À l'écoute des réflexions d'Aimé Guibert: "Le goût, c'est votre musique intérieure. Le goût est quelque chose qui appartient à votre personnalité et qui n'est pas dominant. Le goût vous appartient. Personne ne peut vous empêcher d'aimer quelqu'un ou d'aimer un vin ou un autre. Le goût c'est votre patrimoine. C'est une danse personnelle ", résonnent encore dans l'esprit du spectateur les propos de Robert Parker : «Ce dont je suis très fier, c'est d'avoir apporté un point de vue américain et démocratique à ce breuvage élitiste. C'est surtout en France qu'il y a tant de vieilles familles du vin. Ça remonte à bien avant la Révolution. Ce sont des familles nobles, avec un patrimoine incroyable. Et voilà qu'un américain débarque et dit que vous faites du vin de pique-nique. Ça les déroute».

Mais on ne peut réduire, si tentant soit-il, Mondovino à un épisode, parmi tant d'autres de la relation de pouvoir, qui oppose les vignerons de Bourgogne à la machine Mondavi ${ }^{8}$ ou à des duels Guibert-Rolland ou Montille-Parker dans la mesure où ce film présente précisément des personnages documentaires bien plus nombreux. Entre San Francisco et Bordeaux s'interpose Tolombón, entre Parker et Rolland interfèrent les Columbu qui estiment avoir leur mot à dire.

Nous sommes face à une œuvre qui crée des distances entre les personnages, rapproche des personnages éloignés et éloigne des êtres proches et ce en jouant de "façon raisonnée sur ce que l'on pourrait nommer des variations d'échelles d'observation ${ }^{9}$ ". Dans Mondovino, les locaux côtoient les mondiaux et grâce à la bonne diffusion de ce film, les échos de cette rencontre résonnent à l'échelle mondiale. Ce ne sont plus simplement des images enregistrant des événements dans le temps et l'espace mais des rapports d'images, ce qui n'est pas du tout pareil.

Car que fait un cinéaste devant sa table de montage ? Voici la réponse à cette question de Frederick Wiseman : "Vous bricolez la relation entrer le temps réel et le temps filmé ou monté, et vous bricolez l'ordre des séquences, et vous vous dites qu'elles apparaissent en relation alors qu'elles n'ont en fait aucune relation ni dans le temps ni dans l'espace. Vous êtes en train de créer une fiction avec des matériaux documentaires : ces choses qui apparaissent en relation, peut-être ne sont-elles liées que dans votre esprit. Et la réussite du film dépend de sa capacité globale à créer l'illusion que si le processus, qui vous a amené à la conclusion qu'il y avait effectivement relation, semble avoir une certaine validité au niveau de la forme finale du film ${ }^{10}$ ».

Or, dans Mondovino ce sont précisément les articulations entre le local et le global qui permettent de présenter des réalités sociales distinctes mais pas incompatibles. De la forme même de cette présentation découle une méthode possible pour analyser les mécanismes de la mondialisation.

Non pas la mondialisation des images, non pas la mondialisation dans l'image, mais la mondialisation à travers les images. Des images qui se contaminent, qui transpirent, qui deviennent poreuses et enrichissent l'analyse en nuances, en intensité, en complexité. Le spectateur investit l'image et s'investit, effort de participation au monde, il importe de rappeler que «le hors-champ d'une image n'est plus la scène attenante mais n'importe quelle autre image en circulation au même moment dans la mémoire vive des autres ${ }^{11} »$. Deux réalisateurs avaient pris au sérieux cette dimension du horschamp : Dziga Vertov et Jean-Luc Godard.

Ainsi, nous sommes face à des rapports d'images qui peuvent ébranler la pensée et mettre au défi : défi d'agir ${ }^{12}$, de réagir, de protester, de lutter, d'écouter, de dialoguer, ou au contraire face à des rapports d'images autoritaires, de type hiérarchique qui font naître un malaise, un malaise dans la mondialisation.

En d'autres termes, il me semble que le rôle du chercheur n'est pas de lire la réalité qu'il étudie avec les instruments généralement simplifiants qu'il a reçus mais au contraire de s'ingénier à trouver des instruments à même de créer sans cesse de nouveaux contextes pertinents qui inviteraient à une autre lecture du social. Aborder la mondialisation en mobilisant les dispositifs 
préconisés par le cinéma, ainsi que les théoriciens du montage, m'apparaît l'occasion d'enrichir l'analyse sociale, de la complexifier dans la mesure où des aspects différents, inattendus, multipliés de l'expérience collective sont pris en compte.

Porter son attention de manière spécifique sur les agencements possibles entre les différents contextes pertinents, observer comment s'effectuent ces branchements, analyser les conséquences de ces articulations et combinaisons. Voilà une manière de produire des connaissances anthropologiques propices à appréhender la singularité des phénomènes de mondialisation dans leur pluralité, car comme le rappelle Georges Devereux : "La nature d'une science est complètement définie par son exploitation des aspects de phénomènes que d'autres sciences n'exploitent pas.

Lorsqu'un groupe de sciences s'occupe essentiellement du même phénomène, la discipline qui étudie avec le plus de consistance un aspect de ces phénomènes qui n'est étudié qu'incidemment par les sciences connexes, et pas du tout par les sciences non connexes, sera paradigmatique de ce groupe de sciences ${ }^{13} »$.

Mouloud BoUKaLA

Entretien inédit avec Peter Watkins in La Commune : Paris 1871, D.V.D., La Sept Arte, 2003.

Hubert de Montille est un viticulteur possédant 8 hectares à Volnay et Pommard, en Bourgogne.

Fils du journaliste Bernard Nossiter, Jonathan Nossiter est né aux États-Unis et a grandi en France, en Angleterre, en Italie, en Grèce et en Inde. Après une formation de peinture aux Beaux Arts de Paris et à l'Art Institute de San Francisco, il se lance dans le cinéma. Assistant du réalisateur Adrian Lyne, il réalisera par la suite Resident Alien (1991), Sunday (1997), Signs and Wonders (2000).

4 Ce nouveau procédé consiste à diffuser par intervalles de fines bulles d'oxygène dans les barriques pour faire évoluer plus rapidement le vin, tout en fixant couleurs et tanins. Certains auraient bien aimé que cette technique fût interdite. Elle est en tout cas suffisamment délicate pour faire courir des risques non négligeables au vin, si elle est mal maîtrisée, donnant une lourdeur très caractéristique, aux notes de pruneau et de kirsch.

5 Voici quelques propos tenus par Robert Parker dans le film Mondovino depuis son lieu de résidence, Monkton, dans le Maryland : "Le New York Times peut faire fermer un restaurant. Mais il n'a pas d'effet à Tokyo, ni à Singapour, ni à Paris... Moi, de mon petit bled de Monkton, j'ai un impact mondial... Le « Terroir » en Bourgogne, c'est un code pour une hiérarchie féodale. Cette hiérarchie a permis aux grands Crus d'être vendus plus chers que les Premiers Crus et les Premiers Crus plus chers que les vins d'appellation Village. Moi, c'est le mec qui renverse ce système. Merde. Je m'en fous si c'est un Grand Cru ou si ta famille fait du vin depuis 200 ans. Je m'en fous si tu possèdes cinq putains de châteaux. Ce qui m'importe, c'est ce qu'il y a dans la bouteille. Et tu sauras ce que j'en pense. C'est ça qui dérange... ».

6 Car comme le souligne Bernard Lahire : «À chaque échelle de contexte correspond un ordre de complexité ainsi que des informations pertinentes spécifiques, ordre de complexité et informations pertinentes qui ne sont pas ceux que travaillent d'autres chercheurs à partir d'autres échelles » in « La variation des contextes en sciences sociales ", Annales HSS, mars-avril 1996, n² 2, p. 397.

7 Ces contextes relèvent toujours d'une construction dans laquelle le chercheur tient une place centrale comme le précise C. Jouhaud : «Les contextes n'existent pas préalablement à l'opération qui les construit, ou bien alors il ne s'agit que de présupposés. Il n'y a pas de contextes, mais des opérations, des procédures, des expériences de contextualisation qui touchent de manière essentielle, spécifique et relative une part du réel historique ", in "Présentation ", Annales HSS, mars-avril, n 2, p. 273.

$8 \quad$ Mondavi est la multinationale qui règne dans le monde vinicole. Elle a été fondée par Robert Mondavi en 1966. Cotée au Nasdaq depuis 1993, elle produit 120 millions de bouteilles dans le monde, de Napa au Chili, et de l'Australie à la Toscane.

9 Jacques Revel, «L'histoire au ras du sol », in Le pouvoir au village, Gallimard, Paris, 1989, p. 32.

10 Ira Halberstadt, "An Interview with Fred Wiseman ", in Nonfiction Film, Richard Barsam, Indiana University Press, Indianapolis, 1992, p. 303.

11 Serge Daney, L'exercice a été profitable, Monsieur, P.O.L., Paris, 1993, p. 67.

12 "Si le visuel nous empêche de voir (parce qu'il préfère qu'on décode, qu'on décrypte, bref qu'“on lise" ), l'image nous met toujours au défi de la monter avec une autre, avec de l'autre Parce que dans l'image, comme dans la démocratie, il y a du "jeu " et de l'inachevé, une entame ou une béance ", Serge Daney, Devant la recrudescence des vols de sacs à main, Aléas, Lyon, 1991, p.194.

13 Georges Devereux, De l'angoisse à la méthode, Flammarion, Paris, 1980, p. 400. 


\section{RÉFÉRENCES BIBLIOGRAPHIQUES}

- AMSelle J.L., 2001, Branchements, Flammarion, Paris, Payot.

- Bauman Z., 2004, L’amour liquide, Le Rouergue/Chambon, Rodez.

- Bauman Z., 1999, Le coût humain de la mondialisation, Hachette.

- DANEY S., 1999, Devant la recrudescence des vols de sacs à main, Aléas.

- Daney S., 1993, L'Exercice a été profitable, Monsieur, P.O.L., Paris.

- DevereuX G., 1980, De l'angoisse à la méthode, Flammarion, Paris.

- LAHIRE B., 1996, «La variation des contextes en sciences sociales », Annales HSS, mars-avril 1996, $\mathrm{n}^{\circ} 2$, p.p. 381-407.

- Laplantine F., 2004, « La mondialisation des goûts », Parcours anthropologiques, n 4, p.p. 58-59.

- Revel J., 1989, «L'histoire au ras du sol ", in Le pouvoir au village, Gallimard, Paris, 1989, p.p. 1-33. 